\title{
Study on Payload Effects on the Joint Motion Accuracy of Serial Mechanical Mechanisms
}

\author{
Dan Zhang ${ }^{1}$, Bin $\mathrm{Wei}^{2}$ \\ ${ }^{1}$ Department of Mechanical Engineering, York University, Toronto, Ontario, Canada \\ ${ }^{2}$ Faculty of Engineering and Applied Science, University of Ontario Institute of Technology, Oshawa, Ontario, Canada
}

\begin{abstract}
When the end-effector of the industrial serial mechanical manipulators grasps different payload masses, the output of joint motion will vary, which decreases end-effector positioning accuracy of the overall system. Based on the model reference adaptive control technique, the payload variation effect can be solved and therefore, to improve the positioning accuracy. This paper studies payload effects on the joint motion accuracy of serial mechanical mechanisms.
\end{abstract}

\section{Introduction}

Proportional-integral-derivative (PID) control is the widely used control method in many industries. For example, in many robotic arm used industries, PID control applies to each joint to control the whole robotic arm. By adjusting the PID gains of the PID controller, one can have desired output performance. In [1], a discrete PID controller was designed to employ it in nano scale systems. In [2], the PID controller with additional imposed nonlinear logic was reviewed for robot motion positioning control. In [3], the control strategies that implement planar micro assembly using groups of stressengineered MEMS micro robots controlled through a single global control signal was presented. Model reference adaptive control is another control method that was proposed early by Landau [4] and it has been developed afterwards [5-8]. The reason that one needs to apply the adaptive control, especially the model reference adaptive control approach, is that traditional controllers cannot compensate the payload variations, i.e. when the end-effector grasps different payload masses, the joint output will vary under different payload masses, which will affect the positioning accuracy of the end-effector. Whereas for the model reference adaptive control, the above problem can be effectively resolved and payload variation effect can be compensated. For example, the MRAC (model reference adaptive control) proposed by R. Horowitz and later associated developments by other authors [9] contains an adaptive algorithm block and a position feedback loop which provides the difference between desired and actual position of the joints. This difference is acted upon by the integral portion of a seemingly PID control structure before feedback values of position and velocity are subtracted from it. The problem by using PID controller of not being able to compensate the payload variation is illustrated and the general theory of model reference control is presented in the following section.

\section{Payload Variation Effect and Model Reference Control}

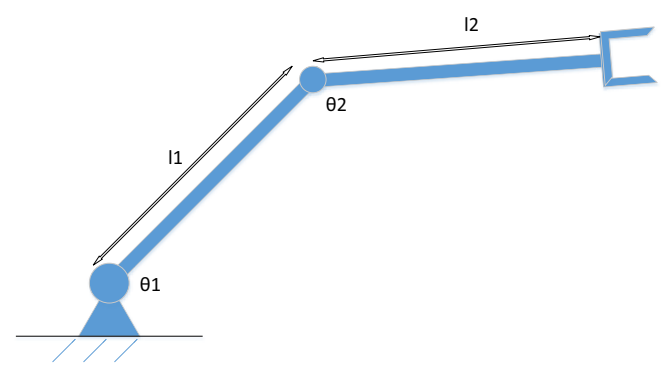

Figure 1. Two-link manipulator

In order to implement PID control of the two-DOF (degrees of freedom) link manipulator case, as shown in Fig. 1, the dynamic equation has to be derived. Here by using the Lagrange method, the torques applied to the joints are determined:

$$
\tau_{1}=\frac{d}{d t} \frac{\partial L}{\partial \dot{\theta}_{1}}-\frac{\partial L}{\partial \theta_{1}}, \tau_{2}=\frac{d}{d t} \frac{\partial L}{\partial \dot{\theta}_{2}}-\frac{\partial L}{\partial \theta_{2}}
$$

The kinetic energy and potential energy for link 1 are expressed as:

$$
K_{1}=\frac{1}{2} m_{1}\left(l_{1} \dot{\theta}_{1}\right)^{2}, P_{1}=m_{1} g\left(l_{1} \sin \theta_{1}\right)
$$


For link 2, first write down the coordinates of the end of link 2, then differentiate them with respect to time in order to obtain the kinetic energy. Denote the Cartesian coordinates of the end of link 2 as $(\mathrm{x} 2, \mathrm{y} 2)$.

$$
\begin{gathered}
x_{2}=l_{1} \cos \theta_{1}+l_{2} \cos \left(\theta_{1}+\theta_{2}\right) \\
y_{2}=l_{1} \sin \theta_{1}+l_{2} \sin \left(\theta_{1}+\theta_{2}\right)
\end{gathered}
$$

Differentiate with respect to time results in:

$$
\begin{gathered}
\dot{x}_{2}=-l_{1} \dot{\theta}_{1} \sin \theta_{1}-l_{2}\left(\dot{\theta}_{1}+\dot{\theta}_{2}\right) \sin \left(\theta_{1}+\theta_{2}\right) \\
\dot{y_{2}}=l_{1} \dot{\theta}_{1} \cos \theta_{1}+l_{2}\left(\dot{\theta}_{1}+\dot{\theta}_{2}\right) \cos \left(\theta_{1}+\theta_{2}\right)
\end{gathered}
$$
as:

Therefore, the kinetic energy for link 2 are expressed

$$
\begin{aligned}
& K_{2}=\frac{1}{2} m_{2} v_{2}^{2}=\frac{1}{2} m_{2} l_{1}^{2} \dot{\theta}_{1}^{2}+\frac{1}{2} m_{2} l_{2}^{2}\left(\dot{\theta}_{1}+\dot{\theta}_{2}\right)^{2} \\
& +m_{2} l_{1} l_{2} \cos \theta_{2} \dot{\theta}_{1}\left(\dot{\theta}_{1}+\dot{\theta}_{2}\right)
\end{aligned}
$$

where $v_{2}^{2}=\dot{x}_{2}^{2}+\dot{y}_{2}^{2}$

The potential energy for link 2 are expressed as:

$$
P_{2}=m_{2} g l_{1} \sin \theta_{1}+m_{2} g l_{2} \sin \left(\theta_{1}+\theta_{2}\right)
$$

The total kinetic and potential energy are expressed as:

$$
\begin{aligned}
& P=P_{1}+P_{2}=\left(m_{1}+m_{2}\right) g l_{1} \sin \theta_{1}+m_{2} g l_{2} \sin \left(\theta_{1}+\theta_{2}\right) \\
& K=K_{1}+K_{2}=\frac{1}{2}\left(m_{1}+m_{2}\right) l_{1}^{2} \dot{\theta}_{1}^{2} \\
& +\frac{1}{2} m_{2} l_{2}^{2}\left(\dot{\theta}_{1}+\dot{\theta}_{2}\right)^{2}+m_{2} l_{1} l_{2} \cos \theta_{2} \dot{\theta}_{1}\left(\dot{\theta}_{1}+\dot{\theta}_{2}\right)
\end{aligned}
$$

The Lagrange is obtained as:

$$
\begin{aligned}
& L=K-P=\frac{1}{2}\left(m_{1}+m_{2}\right) l_{1}^{2} \dot{\theta}_{1}^{2}+\frac{1}{2} m_{2} l_{2}^{2}\left(\dot{\theta}_{1}+\dot{\theta}_{2}\right)^{2} \\
& +m_{2} l_{1} l_{2} \cos \theta_{2} \dot{\theta}_{1}\left(\dot{\theta}_{1}+\dot{\theta}_{2}\right) \\
& -\left(m_{1}+m_{2}\right) g l_{1} \sin \theta_{1}-m_{2} g l_{2} \sin \left(\theta_{1}+\theta_{2}\right)
\end{aligned}
$$

$\tau_{1}=\frac{d}{d t} \frac{\partial L}{\partial \dot{\theta}_{1}}-\frac{\partial L}{\partial \theta_{1}}$

$=\left(\left(m_{1}+m_{2}\right) l_{1}^{2}+m_{2} l_{2}^{2}+2 m_{2} l_{1} l_{2} \cos \theta_{2}\right) \ddot{\theta}_{1}+\left(m_{2} l_{2}^{2}+m_{2} l_{1} l_{2} \cos \theta_{2}\right) \ddot{\theta}_{2}$

$+\left(-2 m_{2} l_{1} l_{2} \sin \theta_{2}\right) \dot{\theta}_{1} \dot{\theta}_{2}+\left(-m_{2} l_{1} l_{2} \sin \theta_{2}\right) \dot{\theta}_{2}^{2}+\left(\left(m_{1}+m_{2}\right) l_{1} \cos \theta_{1}+m_{2} l_{2} \cos \left(\theta_{1}+\theta_{2}\right)\right) g$ $\tau_{2}=\frac{d}{d t} \frac{\partial L}{\partial \dot{\theta}_{2}}-\frac{\partial L}{\partial \theta_{2}}$

$=\left(m_{2} l_{2}^{2}+m_{2} l_{1} l_{2} \cos \theta_{2}\right) \ddot{\theta}_{1}+\left(m_{2} l_{2}^{2}\right) \ddot{\theta}_{2}+\left(m_{2} l_{1} l_{2} \sin \theta_{2}\right) \dot{\theta}_{1}^{2}+m_{2} l_{2} \cos \left(\theta_{1}+\theta_{2}\right) g$

Put them in a matrix form, we can obtain the following:

$$
\left[\begin{array}{l}
\tau_{1} \\
\tau_{2}
\end{array}\right]=M \ddot{\theta}+N+G g=\left[\begin{array}{ll}
m_{11} & m_{12} \\
m_{12} & m_{22}
\end{array}\right]\left[\begin{array}{l}
\ddot{\theta}_{1} \\
\ddot{\theta_{2}}
\end{array}\right]+\left[\begin{array}{l}
n_{11} \\
n_{21}
\end{array}\right]+\left[\begin{array}{l}
g_{11} \\
g_{21}
\end{array}\right] g
$$

where

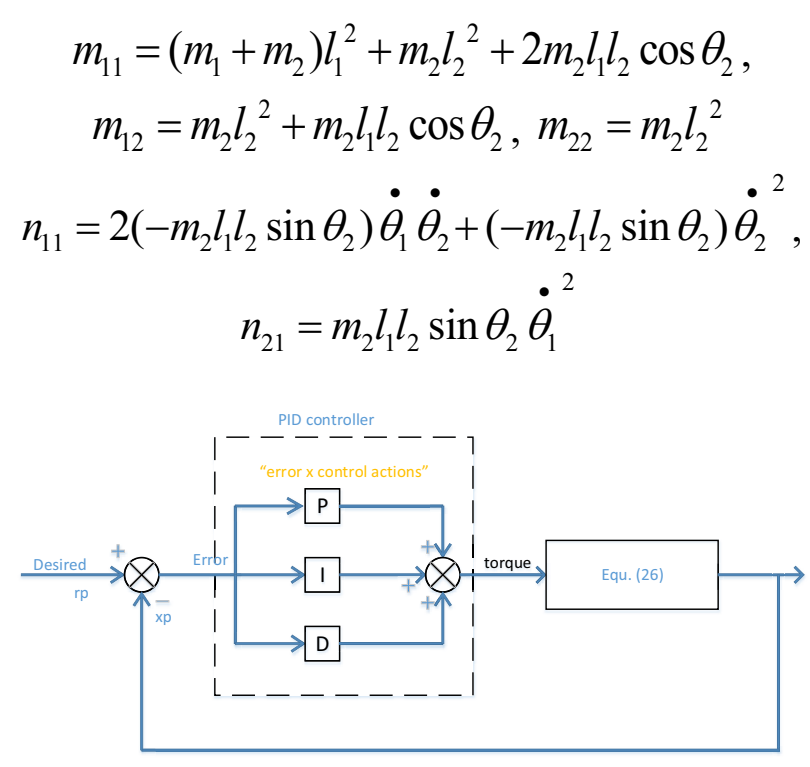

Figure 2. PID control of two-link manipulator

Now apply the PID controller, as shown in Fig. 2, the controller output is the torque, i.e.

$$
K_{p} e+K_{i} \int e d t+K_{d} \dot{e}=\left[\begin{array}{l}
\tau_{1} \\
\tau_{2}
\end{array}\right]
$$

where error $e=r_{p}-x_{p}$

We know the two-link manipulator $\mathrm{M}$ and $\mathrm{N}$ matrices, the output from the manipulator (i.e. acceleration of the joints 1 and 2) can be determined as follows:

$$
\left[\begin{array}{l}
\tau_{1} \\
\tau_{2}
\end{array}\right]=M \ddot{\theta}+N+G g=\left[\begin{array}{ll}
m_{11} & m_{12} \\
m_{12} & m_{22}
\end{array}\right]\left[\begin{array}{c}
\ddot{\theta}_{1} \\
\ddot{\theta}_{2}
\end{array}\right]+\left[\begin{array}{l}
n_{11} \\
n_{21}
\end{array}\right]+\left[\begin{array}{l}
g_{11} \\
g_{21}
\end{array}\right] g
$$

So

$$
\begin{aligned}
& K_{p} e+K_{i} \int e d t+K_{d} \dot{e}=\left[\begin{array}{c}
\tau_{1} \\
\tau_{2}
\end{array}\right]=M \ddot{\theta}+N+G g \\
& \Rightarrow\left[\begin{array}{c}
\ddot{\theta_{1}} \\
\ddot{\theta_{2}}
\end{array}\right]=M^{-1}\left(K_{p} e+K_{i} \int e d t+K_{d} \dot{e}-N\right)
\end{aligned}
$$

After deriving the acceleration of joints 1 and 2, take the time integral to obtain the velocity of joints 1 and 2 and take another integral to obtain the positon of joints 1 and 2 .

$$
\left[\begin{array}{c}
\dot{\theta}_{1} \\
\dot{\theta_{2}}
\end{array}\right]=\int\left[\begin{array}{c}
\ddot{\theta}_{1} \\
\ddot{\theta_{2}}
\end{array}\right] d t,\left[\begin{array}{l}
\theta_{1} \\
\theta_{2}
\end{array}\right]=\int\left[\begin{array}{c}
\dot{\theta}_{1} \\
\dot{\theta}_{2}
\end{array}\right] d t
$$



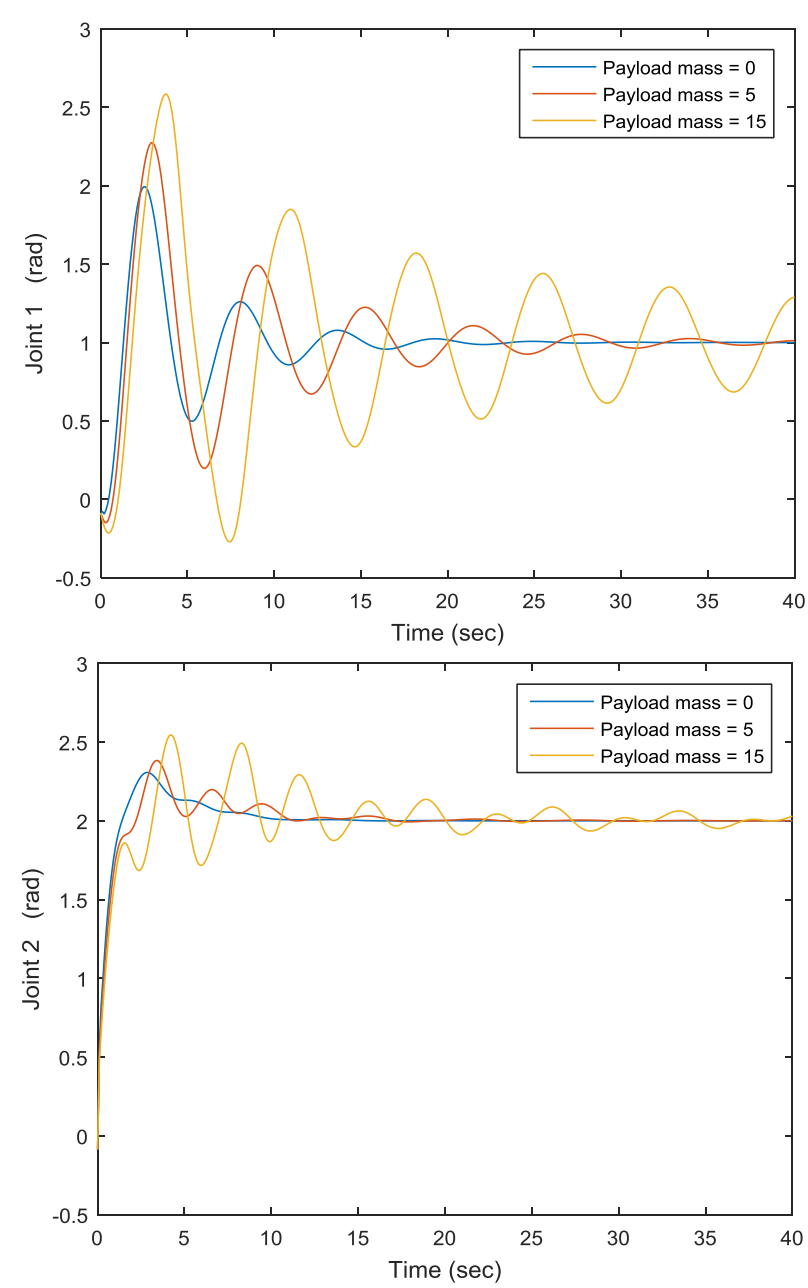

Figure 3. Joints 1 and 2 output

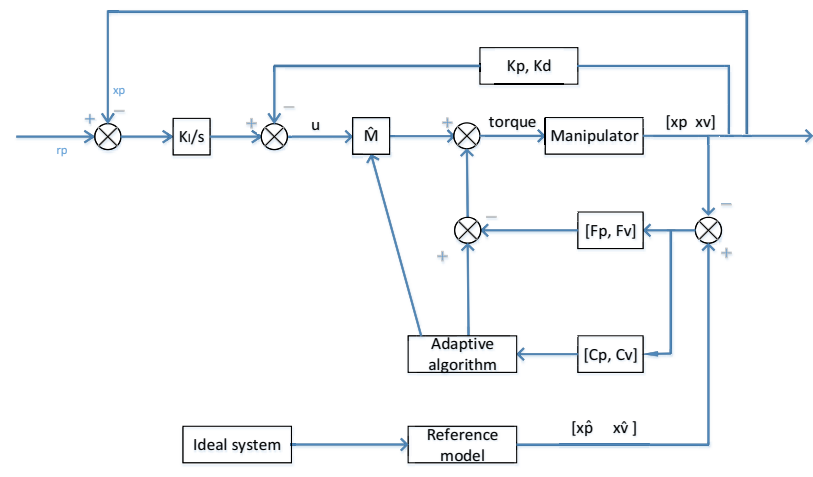

Figure 4. Model reference control approach

After applying different payload masses, the joints motion output are illustrated in Fig. 3. For the joint 1, when the payload is 0 , joint one motion is quite steady, but when the payload increases to 5 and 15 , one can see that joint 1 motion is not the same anymore, and also the joint output is going up and down as shown in Fig. 8. Same applies to joint 2 . In order to address the above problem, model reference control is applied to compensate the payload variation effect. Fig. 4 shows a model reference control approach. Similarly with the PID control, the output from the controller can be determined as follows. For the model reference adaptive control approach,

$$
\text { ControllerOut }=\tau=\hat{M} u+\hat{V}-F_{p} e-F_{v} \dot{e}
$$

where $u=K_{I} \int\left(r_{p}-x_{p}\right)-K_{p} x_{p}-K_{d} x_{v}$

The manipulator dynamic equation is:

$$
\tau=M a+V+G g
$$

So the output from the manipulator (i.e. acceleration of joint) is:

$$
\begin{gathered}
\hat{M} u+\hat{V}-F_{p} e-F_{v} \dot{e}=\tau=M a+V \\
\Rightarrow\left[\begin{array}{l}
a_{1} \\
a_{2}
\end{array}\right]=\left[\begin{array}{c}
\ddot{\theta}_{1} \\
\ddot{\theta_{2}}
\end{array}\right]=M^{-1}\left(\hat{M} u+\hat{V}-F_{p} e-F_{v} \dot{e}-V\right)
\end{gathered}
$$

After deriving the acceleration of joint, take the time integral to obtain the velocity of joint and take another integral to obtain the positon of joint. The adaptive algorithm is derived as follows:

$$
\begin{aligned}
\int_{0}^{T} y^{T}(t) \tilde{M} u(t) d t & =\int_{0}^{T}\left[\begin{array}{l}
y_{1} \\
y_{2}
\end{array}\right]^{T}\left[\begin{array}{cc}
\tilde{m}_{11} & \tilde{m}_{12} \\
\tilde{m_{12}} & \tilde{m}_{22}
\end{array}\right]\left[\begin{array}{l}
u_{1} \\
u_{2}
\end{array}\right] d t \\
& =\int_{0}^{T}\left[y_{1}, y_{2}\right]\left[\begin{array}{cc}
\tilde{m_{11}} & \tilde{m}_{12} \\
\tilde{\tilde{m}} & \tilde{m}_{22} \\
m_{12} & m_{22}
\end{array}\right]\left[\begin{array}{l}
u_{1} \\
u_{2}
\end{array}\right] d t \\
& =\int_{0}^{T} \tilde{m_{11}} y_{1} u_{1} d t+\int_{0}^{T} \tilde{m}_{12}\left(y_{1} u_{2}+y_{2} u_{1}\right) d t+\int_{0}^{T} \tilde{m}_{22} y_{2} u_{2} d t
\end{aligned}
$$

Consider the first term in above equation, we need to find $\frac{d}{d t} \hat{m_{11}}(t)=\frac{d}{d t} \hat{m}_{11}(t)$, so that $\int_{0}^{T} \approx \tilde{m}_{11} y_{1} u_{1} d t \geq-\gamma^{2}$.

From

$\int_{0}^{T} z(t)^{T} z \dot{(t)} d t=\frac{z(T)^{T} z(T)}{2}-\frac{z(0)^{T} z(0)}{2} \geq-\frac{z(0)^{T} z(0)}{2}=-\gamma_{0}^{2}$

So by selecting $\frac{d}{d t} \hat{m}_{11}(t)=\frac{d}{d t} \tilde{m}_{11}(t)=k_{m 11} y_{1} u_{1}$

$$
\Rightarrow y_{1} u_{1}=\frac{m_{11}(t)}{k_{m 11}}
$$

Then

$\int_{0}^{T} \approx \tilde{m}_{11} y_{1} u_{1} d t=\int_{0}^{T} \approx \stackrel{\bullet}{\approx} \tilde{m}_{11} \tilde{m}_{11} \frac{1}{k_{m 11}} d t=\frac{1}{k_{m 11}} \int_{0}^{T} \underset{m_{11}}{\approx} \stackrel{\bullet}{m_{11}} d t \geq-\gamma^{2}$

Using the same analysis on the other two terms, we obtain

$$
\begin{gathered}
\frac{d}{d t} \hat{m_{12}}(t)=\frac{d}{d t} \hat{m_{12}}(t)=k_{m 12}\left(y_{1} u_{2}+y_{2} u_{1}\right) \\
\frac{d}{d t} \hat{m_{22}}(t)=\frac{d}{d t} \approx m_{22}(t)=k_{m 22} y_{2} u_{2}
\end{gathered}
$$


Derivation for $\mathrm{M}$ has finished, now using the same approach, we can obtain the adaptive algorithm for $\mathrm{N}$ as follows:

$$
\begin{gathered}
\frac{d}{d t} \hat{n_{12}}(t)=\frac{d}{d t} \approx \tilde{n_{12}}(t)=k_{n 12}\left(2 y_{1} x_{v 1} x_{v 2}-y_{2} x_{v 1}{ }^{2}\right) \\
\frac{d}{d t} \hat{n_{22}}(t)=\frac{d}{d t} \hat{n_{22}}(t)=k_{n 22} y_{1} x_{v 2}{ }^{2}
\end{gathered}
$$
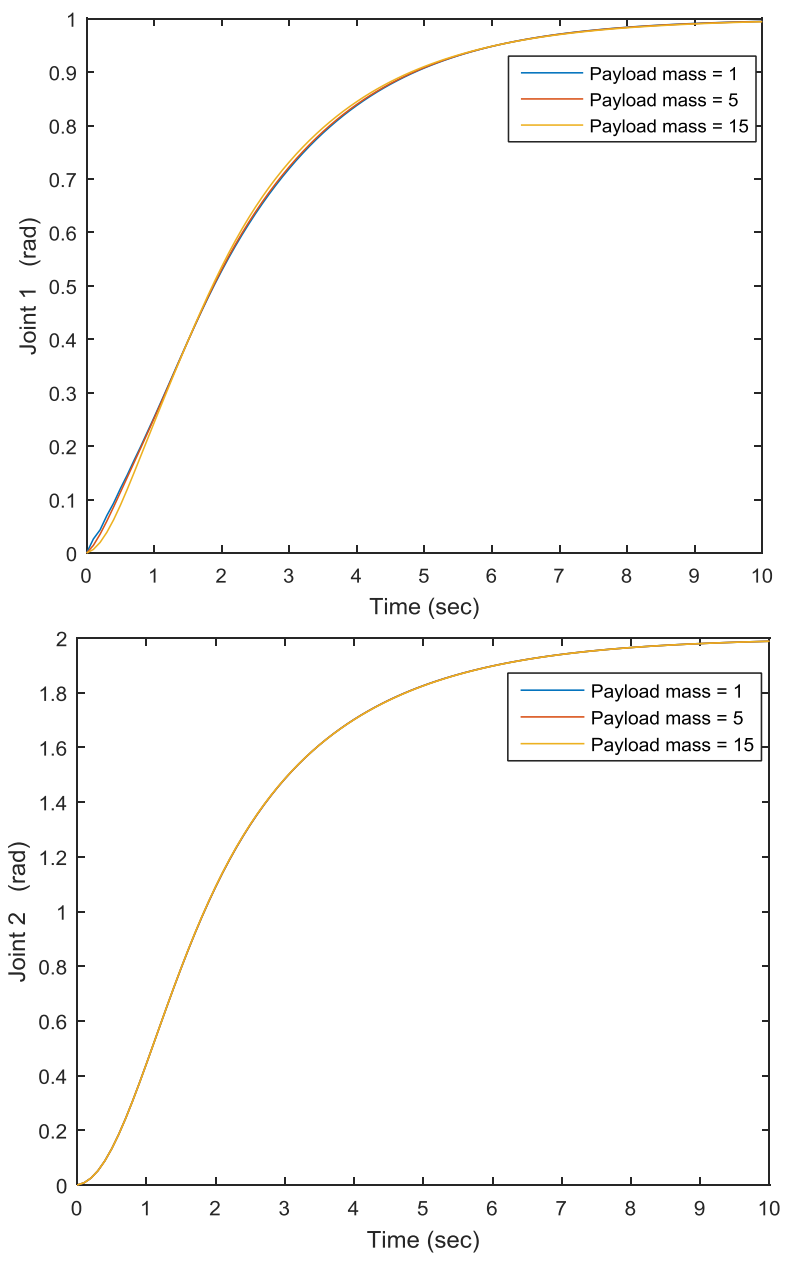

Figure 5. Joints 1 and 2 output

Fig. 5 shows the joints output that under different payload masses. By using the model reference adaptive control approach, three line coincide with each other under different payload masses, i.e. the payload mass variation effect has been compensated.

\section{Conclusion}

When the end-effector grasps different payload masses, the output of joint motion will vary, which will decrease the positioning accuracy of the end-effector. Based on the model reference adaptive control approach, the payload variation effect can be solved effectively and therefore, to improve the positioning accuracy. Future research will focus on hybrid control design by combining model reference adaptive control and PID control for serial robotic arms.

\section{Acknowledgement}

The authors would like to thank the financial support from the Natural Sciences and Engineering Research Council of Canada (NSERC) and Canada Research Chairs program.

\section{References}

1. Navid Razmjooy and Mehdi Ramezani, Using Quantum Gates to design a PID Controller for Nano robots. International Research Journal of Applied and Basic Sciences, Vol, 8 (12): 2354-2359, 2014.

2. Soukeyna Bouchebout, Aude Bolopion, Jean-Ochin Abrahamians, Stephane Regnier. An overview of multiple DoF magnetic actuated micro-robots. Journal of Micro-Nano Mechatronics, 7 (4), pp.97113. 2012

3. Bruce $\mathrm{R}$ Donald, Christopher G Levey, Igor Paprotny and Daniela Rus. Planning and control for micro assembly of structures composed of stressengineered MEMS micro robots. The International Journal of Robotics Research, 32(2), pp. 218-246, 2013.

4. Landau, Y. D., Adaptive control: the model reference approach, Marcel Dekker, New York, 1979.

5. S. Dubowsky, D. Desforges, The application of model-referenced adaptive control to robotic manipulators, Journal of Dynamic Systems Measurement and Control, Vol. 101, (1979) 193-200.

6. Cao, C. and Hovakimyan, N., Design and Analysis of a Novel L1 Adaptive Control Architecture with Guaranteed Transient Performance, IEEE Transactions on Automatic Control, Vol. 53, No. 2, (2008) pp. 586-591.

7. Priyank Jain and Dr. M.J. Nigam. Design of a Model Reference Adaptive Controller Using Modified MIT Rule for a Second Order System. Advance in Electronic and Electric Engineering, Volume 3, Number 4 (2013) pp. 477-484.

8. Nhan T. Nguyen, Kalmanje Krishnakumar, Jovan Boskovic. An Optimal Control Modification to Model-Reference Adaptive Control for Fast Adaptation. AIAA Guidance, Navigation and Control Conference and Exhibit, August (2008) Honolulu, Hawaii. 1-19.

9. R. Horowitz, M. Tomizuka, An Adaptive Control Scheme for Mechanical ManipulatorsCompensation of Nonlinearity and Decoupling Control, Journal of Dynamic Systems, Measurement, and Control, Volume 108, (1986) Issue 2, 1-9. 